\title{
Multidisciplinary Design and Collaborative Optimization for Excavator Backhoe Device
}

\author{
Yin Guang-qiu ${ }^{1,2}$, Lin Shu-wen ${ }^{2}$ and Perlurst, D.B. ${ }^{3}$ \\ ${ }^{1}$ School of Mechanical and Automotive Engineering, Fujian University of Technology, 350118, Fujian, China \\ ${ }^{2}$ School of Machine Engineering and Automation, Fuzhou University, 350106, Fujian, China \\ ${ }^{3}$ Division of Mechanical Engineering, Advanced Machine Engineering and Automation Center, Singapore 121986, Singapore
}

Received 15 March 2014; Accepted 10 July 2014

\begin{abstract}
The excavator working device is a typical mechanical system of electromechanical liquid that is complex. Traditional optimization design methods are difficult to get global optimized results of excavator backhoe device through the serial mode of "mechanism-load-structure". Thus, the theory of parallel collaborative optimization (CO) is applied. To establish a sophisticated $\mathrm{CO}$ model of the backhoe device, a certain excavator is investigated as a sample multidisciplinary $\mathrm{CO}$ (MDCO) design. To generate the $\mathrm{CO}$ model, an improved optimization algorithm called the particle swarm-genetic algorithm (PS-GA) is proposed. To verify the MDCO design of the excavator backhoe device, a parameterized virtual prototype (VP) of the backhoe device is established in ADAMS. This VP is optimized by applying the MDCO design results to the parameterized VP. The VP of the backhoe device is also optimized by a single discipline when the optimization results from a single discipline are inputted into the parameterized VP. Both optimized VPs are simulated under similar conditions, and results show that in the MDCO design, the arm crowd force of the backhoe device is $8.1 \%$ stronger than that in the design optimized by a single discipline under constant power and oil pressure conditions. Similarly, breakout force increased by approximately $8.3 \%$. The quality (volume) of the entire backhoe device decreased by $9.5 \%$; however, the maximum stress of each characteristic partition changed only slightly. Therefore, the MDCO design effectively and practically addresses problems regarding the optimization of the design of complex mechanical systems.
\end{abstract}

Keywords: collaborative optimization, multiple disciplines, backhoe device, global optimization

\section{Introduction}

Collaborative optimization (CO) is a strategy of multidisciplinary design optimization (MDO). It was proposed by Kroo [1] and generally consists of a bi-level optimization structure. This structure not only benefits the organization of a complex system for optimization, but also promotes disciplinary autonomy while maintaining interdisciplinary compatibility. With this architecture, $\mathrm{CO}$ effectively optimizes the design of a complex system in practical engineering and is currently considered a practical and effective method to address problems in the optimization of complex systems [2].

The excavator working device is a typical mechanical system of electromechanical liquid that is complex [3]. It contains various design parameters such as mechanism and structure parameters. In the optimization of the working device, these parameters are difficult to determine because they influence, restrict, and integrate with one another. The current design process of excavator working equipment mainly follows this order: "the mechanism parameters of the working device are designed first. Dynamic analysis is then conducted. The load of the main components is determined,

\footnotetext{
*E-mail address: ylkeeper@163.com
}

ISSN: 1791-2377 @ 2014 Kavala Institute of Technology. All rights reserved. and finally, the structural parameters of the main components are designed". In the calculation order of "mechanism-load-structure", the mechanism establishes the load, and the load determines the structure without a followup calculation. Thus, optimization design is limited to a single discipline (SDO), and the interactions among various disciplines are not considered. Hence, the SDO design is not global.

With respect to the working device, the design optimization order "mechanism-load-structure" not only requires optimizations from disciplines at each stage, but it must also be optimized as a whole simultaneously. Global optimization is multilevel, and its goal differs from that of disciplinary optimization. Thus, we must ensure that the disciplinary optimization goal is met given the requirement for global optimization. MDO is a research topic that has been examined as a design problem and from the perspective of design structure and information organization. In MDO, the design calculation framework, which is helpful to integrate optimization algorithms, is proposed [4]. This framework organically integrates disciplinary knowledge into optimization methods to formulate an effective optimization algorithm, which can be used to optimize complicated objects globally, in terms of disciplines, and component design for complex systems [5], [6]. 
This paper is organized as follows: In Section 2, an improved PSO algorithm is proposed, and the multidisciplinary $\mathrm{CO}(\mathrm{MDCO})$ model of an excavator backhoe device model is established and discussed. In Section 3, the MDCO model of the backhoe device is determined using the enhanced PSO algorithm. The computed results are then discussed in Section 4. Finally, conclusions are drawn in Section 5.

\section{CO Model of the Design Process of the Backhoe Device}

\subsection{Problem of Serial Process Design}

In a sequential design process, feedback is limited. Thus, the flow of information between disciplines is one-way, and the design can only be optimized within the current discipline. Hence, the disciplinary optimal does not correspond to the global optimal. In the global optimization of the excavator working device, therefore, we must consider the mechanism, load, and structure of the working equipment. The design process must shift from a unidirectional sequential pattern into a multidirectional circular pattern, as shown in Fig. 1. As a result, the calculated amount increases sharply, and convergence is uncertain. Coordination between global optimization and disciplinary optimization is induced by the CO design process, which disturbs the couplings among disciplines by introducing equality constraints in system layer. Thus, each disciplinary optimization autonomously focuses on only its own constraints. These design optimizations are parallel, as shown in Fig. 2. With this method, global optimization of large and complex systems can be easily achieved.

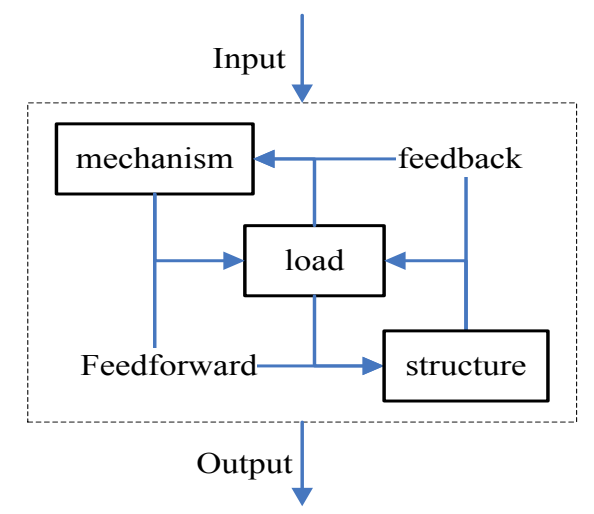

Fig. 1 Feedback of Serial Design

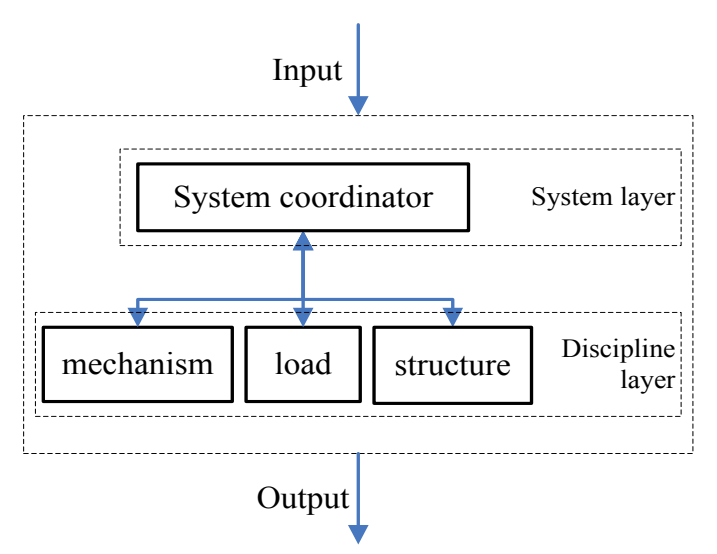

Fig. 2 CO Hierarchical Structure.

\subsection{Parallel MDCO Model}

\subsubsection{CO Principle}

The distributed $\mathrm{CO}$ method with bi-level optimization structure was proposed by Kroo [1] and is used in multiobjective MDO. In this method, the optimization problem of complex system design is decomposed into different disciplinary design optimizations and system-level optimization problems. The design variables shared by various disciplines are coordinated by the equality constraints at system level, as are the variables of coupling state. The basic CO framework is depicted in Fig. 3. This framework is divided into two main parts: system level optimization (global optimization) and subsystem optimizations (disciplinary optimization). The design variables in subsystem optimization include shared design variables, coupling variables at disciplinary state, and local variables of disciplinary design. In the disciplinary optimization process, each discipline is required to meet only its own constraints. Subsystem optimization aims to differentiate its goal from that of the system level at minimum. Thus, system level optimization targets the entire system. Given the equality constraint condition, the variables of interdisciplinary design and coupling state remain constant.

Each subsystem can parallel analysis and optimiza-tion. Therefore, complex system analysis can be eliminated in this method. The goal of subsystem optimization is not directly related to the target value of the entire system. However, the state variables serve as design variables, and the dimensions of these design variables expand. Thus, the number of subsystems analyzed increases during $\mathrm{CO}$, and the iterative convergence is gradual. Furthermore, equality constraints are difficult to meet at system level. To simplify this process, the equation constraints are transformed into inequality constraints using the slack variable method. These problems have been investigated in numerous previous studies [7],[8],[9].

To compute the MDCO model, intelligent group algorithms [i.e., genetic algorithm(GA), particle swarm optimization (PSO), and ant colony optimization (ACO) algorithms] are often used. However, a single intelligent group algorithm is inadequate to address the MDCO model for a complex system. Some of these algorithms can generate a feasible solution, but not the global optimal one [10]. Thus, this study proposes an effective particle swarmgenetic algorithm (PS-GA).

\subsubsection{PS-GA}

The MDCO model of a complex system contains both continuous and discrete design variables. It has many optimization goals and complex constraint functions. This design optimization problem is nonlinear and integrates continuous and discrete variables. This issue is difficult to address using traditional methods, including GA, PSO, and ACO algorithms. Therefore, this study develops an improved PSO algorithm that essentially incorporates the hybrid concept of GA into the PSO algorithm to obtain a composite intelligent group algorithm. The principle of the algorithm is as follows: Using the hybrid concept in GA, we select a specific number of particles from the hybrid pool based on the hybrid probability in each iteration. In the pool, two particles form a random hybrid and produce similar amounts of offspring particles (son). The offspring then replace their parent particles (father) to accelerate the 
evolution of the particle swarm and improve solving efficiency. GA complements the algorithm based on PSO;

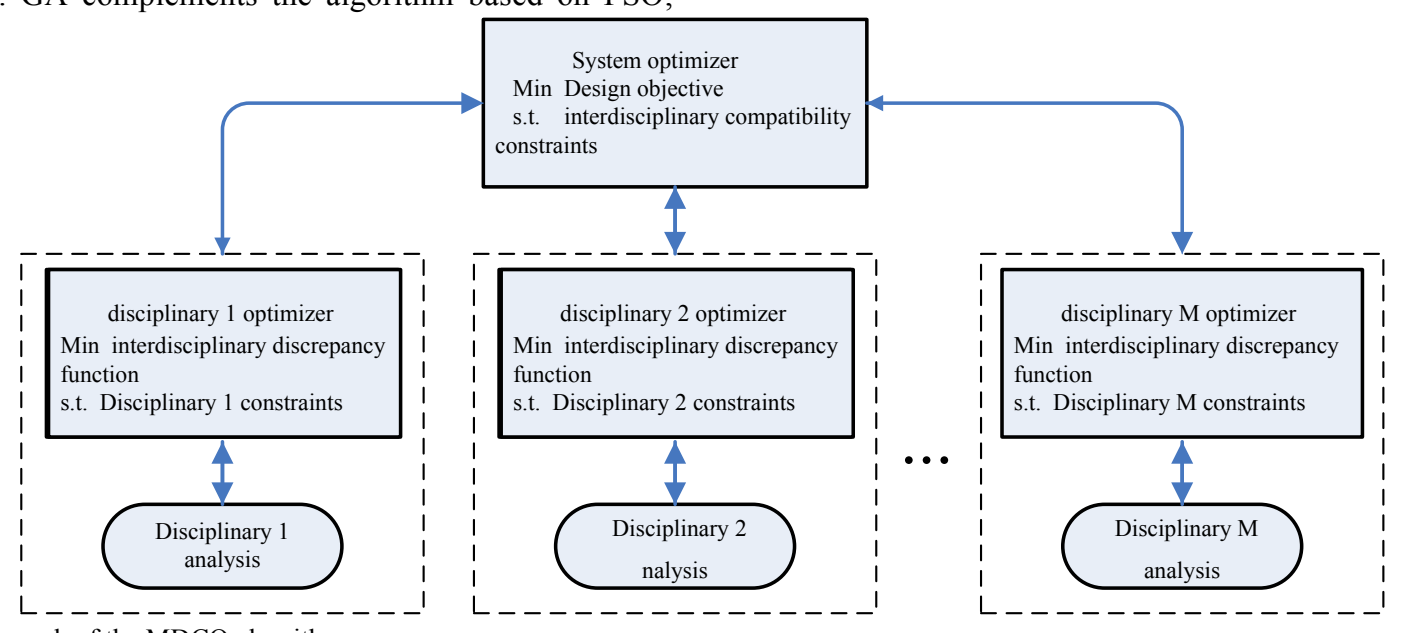

Fig. 3 Framework of the MDCO algorithm

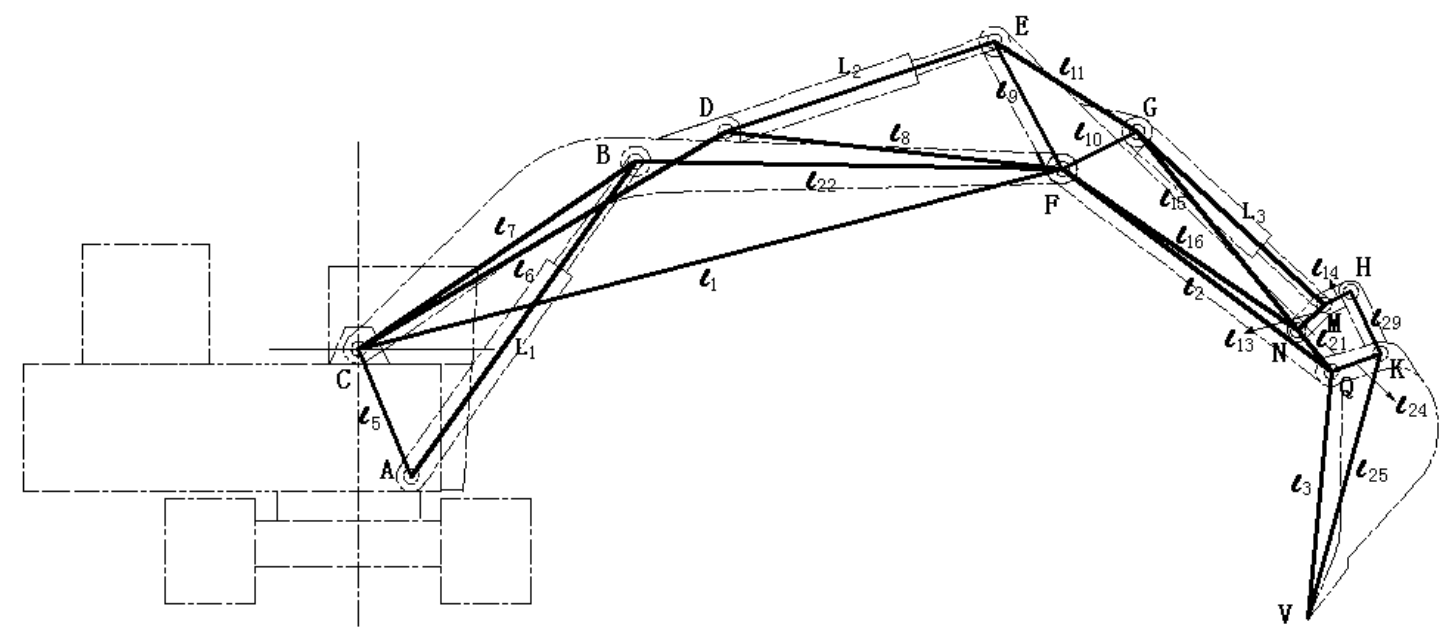

Fig. 4 Mechanism model of the excavator backhoe device

Hence the label PS-GA. To obtain the location of the offspring given the parent location, an arithmetic crossover is applied as follows (1):

$$
\operatorname{son}(x)=\lambda \cdot \operatorname{father}_{1}(x)+(1-\lambda) \cdot \text { father }_{2}(x)
$$

Where $\lambda \in[0,1]$, random number. (2):

The speed of the offspring is calculated using Formula

$$
\operatorname{son}(v)=\frac{\mid \text { father }_{1}(v) \mid}{\mid \text { father }_{1}(v)|+| \text { father }_{2}(v) \mid}\left(\text { father }_{1}(v)+\text { father }_{2}(v)\right)
$$

or

$$
\operatorname{son}(v)=\frac{\mid \text { father }_{2}(v) \mid}{\mid \text { father }_{1}(v)|+| \text { father }_{2}(v) \mid}\left(\text { father }_{1}(v)+\text { father }_{2}(v)\right)
$$

The basic steps in the PS-GA are as follows:

1) In the population, the position and velocity of each particle are initialized at random.

2) The fitness value of each particle is evaluated. It is stored in the pbest variable of each particle along with current position. If a particle displays an optimal fitness value, it is included in the gbest variable along with its current position.

3) The speed and position of each particle are updated.

4) The fitness value of each particle is compared with that of the ideal position based on experience. The pbest variable is then updated if the fitness value of the study particle is higher.
5) All of the current pbest values are compared with those of gbest. gbest is then updated if the pbest values are superior.

6) According to the hybridization probability, a specified number of parent particles are selected from the hybrid pool. In the pool, two particles form a random hybrid and generate the same number of offspring particles. The positions and velocities of the offspring particles are calculated using Formulas (1) and (2). The values of gbest and pbest remain unchanged.

7) If the stop condition (i.e., the preset number of calculation precision or iterations) is reached, the search ceases and the output is printed. Otherwise, the algorithm returns to step 3 and continues the search.

\subsection{MDCO Model for the Backhoe Device}

\subsubsection{Model to Optimize Mechanism}

The mechanism model for the excavator backhoe device is presented in Fig. 4. In the backhoe device, the design mechanism determines the operating range (maximum reach, digging depth, and dumping height), digging force, and the matching degree of digging resistance. These factors influence the working efficiency of the backhoe device.

Mechanism optimization aims to match the degrees of digging force and resistance. In the design optimization of 
the backhoe device, the distances between hinge points are used as variables. With respect to mechanism, the optimization constraints are the operating range, motion performance, and geometry conditions. During excavation, bucket and arm digging are typically utilized. Thus, the digging force and resistance are matched as follows:

(1) During arm excavation, optimization aims to match arm crowd force and digging resistance. Within the domain, the optimization model of the backhoe device is determined as follows:

$$
\begin{aligned}
& \text { Find } \quad X_{1} \\
& \min \delta_{1}\left(X_{1}\right)=\sum_{i=1}^{4} \sum_{j=1}^{20}\left(F_{i j}\left(X_{1}\right)-W_{i j}\left(X_{1}\right)\right)^{2} \\
& \text { s.t. } \quad g_{i}\left(X_{1}\right) \leq 0
\end{aligned}
$$

where

$$
\begin{gathered}
X_{1}=\left[\begin{array}{lllllllll}
l_{1}, & l_{3}, & l_{6}, & l_{8}, & l_{9}, & l_{10}, & l_{11}, & l_{13}, & l_{14}, \\
l_{15}, & l_{16}, & l_{21}, & l_{22}, & l_{25} & L_{1 \max }, & L_{1 \min }, & L_{2 \max }, \\
L_{2 \min }, & L_{3 \max }, & L_{3 \min }, & K_{1}, & K_{2}, & \sigma
\end{array}\right]^{T}
\end{gathered}
$$

denotes the length between the hinges and characteristic parameters; $F_{i j}\left(X_{1}\right)$ is arm crowd force during arm excavation, $W_{i j}\left(X_{1}\right)$ is arm digging resistance during arm excavation, and $g_{i}\left(X_{1}\right)$ represents the constraints of working space and mechanism motion performance in the backhoe device, as well as the composite conditions of the mechanism.

(2) During bucket excavation, optimization aims to match breakout force and the resistance to bucket digging. Within the domain, the optimization model of the backhoe device is expressed as follows:

$$
\begin{aligned}
& \text { Find } \quad X_{2} \\
& \min \delta_{2}\left(X_{2}\right)=\sum_{i=1}^{4} \sum_{j=1}^{20}\left(P_{i j}\left(X_{2}\right)-Q_{i j}\left(X_{2}\right)\right)^{2} \\
& \text { s.t. } \quad g_{i}\left(X_{2}\right) \leq 0 \\
& \text { Where } \\
& X_{2}=\left[\begin{array}{llllllllll}
l_{1}, & l_{3}, & l_{6}, & l_{8}, & l_{9}, & l_{10}, & l_{11}, & l_{13}, & l_{14}, \\
l_{15}, & l_{16}, & l_{21}, & l_{22}, & l_{25} & L_{1 \max }, & L_{1 \min }, & L_{2 \max }, \\
L_{2 \min }, & L_{3 \max }, & L_{3 \min }, & K_{1}, & K_{2}, & \sigma
\end{array}\right]^{T}
\end{aligned}
$$

denotes the length between the hinges and characteristic parameters, $F_{i j}\left(X_{2}\right)$ is the breakout force during bucket excavation; $W_{i j}\left(X_{2}\right)$ is the bucket digging resistance during bucket excavation; $g_{i}\left(X_{2}\right)$ denotes the constraints of working space and mechanism motion performance, as well as the composite conditions of the mechanism.

\subsubsection{Model to Optimize Structure}

The backhoe device is mainly composed of the boom, arm, and bucket. The structures of these components are exhibited in Fig. 5.

In structural design, shape and size are determined. Moreover, the weight, structure shape, and connection mode of the backhoe device are key in structure optimization because they influence digging force and external load distribution in different parts of the backhoe device. In the structural optimization of the backhoe device, the structure parameter $X_{3}$ is the design variable. To meet the constraint conditions of static and fatigue strength and stiffness, optimization aims to minimize the volume of the backhoe device. Thus, the mathematical model of the structural optimization of the backhoe device is expressed as:

$$
\begin{aligned}
& \text { Find } \quad X_{3} \\
& \min V\left(X_{3}\right)=\sum_{i=1}^{3} V_{i}\left(X_{3}\right) \\
& \text { s.t. } \quad g_{i}\left(X_{3}\right) \leq 0
\end{aligned}
$$

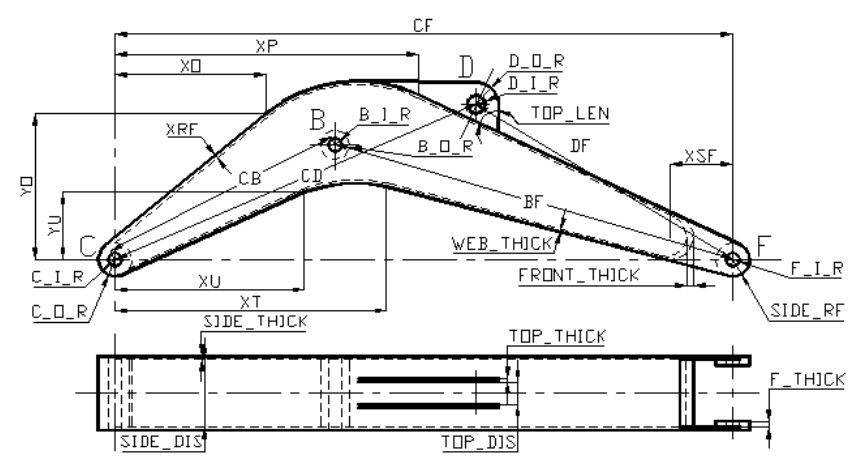

a)

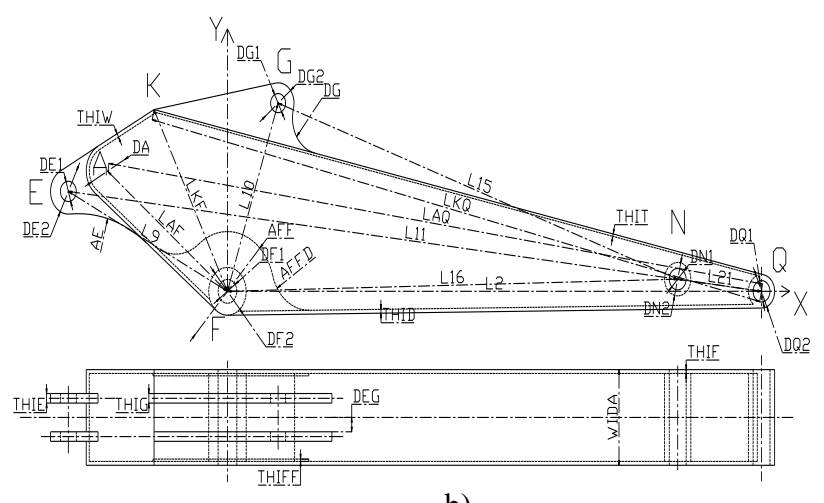

b)

Fig. 5 Structure diagrams of (a) the boom and (b) the arm

Where

$X_{3}=\left[S I D E \_T H I C K, \quad Y U, \quad S I D E_{-} D I S, \quad X R F\right.$,

$W E B \_T H I C K, \quad X T, \cdots, X O, \quad B_{-} O_{-} R$,

$\left.C_{-} O_{-} R, \quad S I D E_{-} R F, \quad T O P_{-} T H I C K, \quad \cdots \quad D_{-} O_{-} R\right]^{T}$

indicates the detailed structure parameters of each main component, $V_{i}\left(X_{3}\right)$ represents the volumes of the boom, arm, and bucket; $g_{i}\left(X_{3}\right)$ denotes the constraints of strength, stiffness, and fatigue strength.

\subsubsection{MDCO Model}

In the various models of disciplinary optimization mentioned above, the shared variables are the coupling variables in each disciplinary optimization. Hence, the values of these variables may vary. This occurrence can be attributed to the CO process, wherein the values of the shared and coupling variables change to benefit the disciplinary optimization of each model. This variation is guided by the disciplinary optimization goals and is restricted by the constraint conditions of each discipline. To reconcile the values of the shared and coupling variables, each disciplinary optimization model must compromise and concede. With the CO strategy, the target value of individual disciplinary optimizations is lower than that of each independent goal in disciplinary optimization. Therefore, $\mathrm{CO}$ aims to generate the optimal value for each independent discipline and to obtain results that are close to those of independent optimization. If the independent target value of individual disciplinary optimization is defined as the ideal point, the optimal results obtained by $\mathrm{CO}$ must match the independent 
value of each disciplinary optimization given that the values of the shared and coupling variables are consistent. Hence, the sophisticated CO model can be abstracted as follows: Find $\bar{X} \quad \bar{Y}$

$\min \left(\left(\delta_{1}\left(X_{1}\right)-\delta_{1}^{*}\right) / \delta_{1}^{*}\right)^{2}+\left(\left(\delta_{2}\left(X_{2}\right)-\delta_{2}^{*}\right) / \delta_{2}^{*}\right)^{2}+\left(\left(V\left(X_{3}\right)-V^{*}\right) / V^{*}\right)^{2}$ s.t. $\quad \bar{X}_{\text {min }} \leq \bar{X} \leq \bar{X}_{\text {max }} \quad \bar{Y}_{\min } \leq \bar{Y} \leq \bar{Y}_{\text {max }}$

where $\bar{X}$ represents the shared variables; $\bar{X}=X_{1} \cap X_{2} \cap X_{3}$, $\bar{Y}$ denotes the variable of coupling state; and $\delta_{1}^{*}, \delta_{2}^{*}, V^{*}$ correspond to the independent target values of disciplinary optimization.

Given the observations above, the $\mathrm{CO}$ model of the backhoe device can be detailed as shown in Fig. 6 .

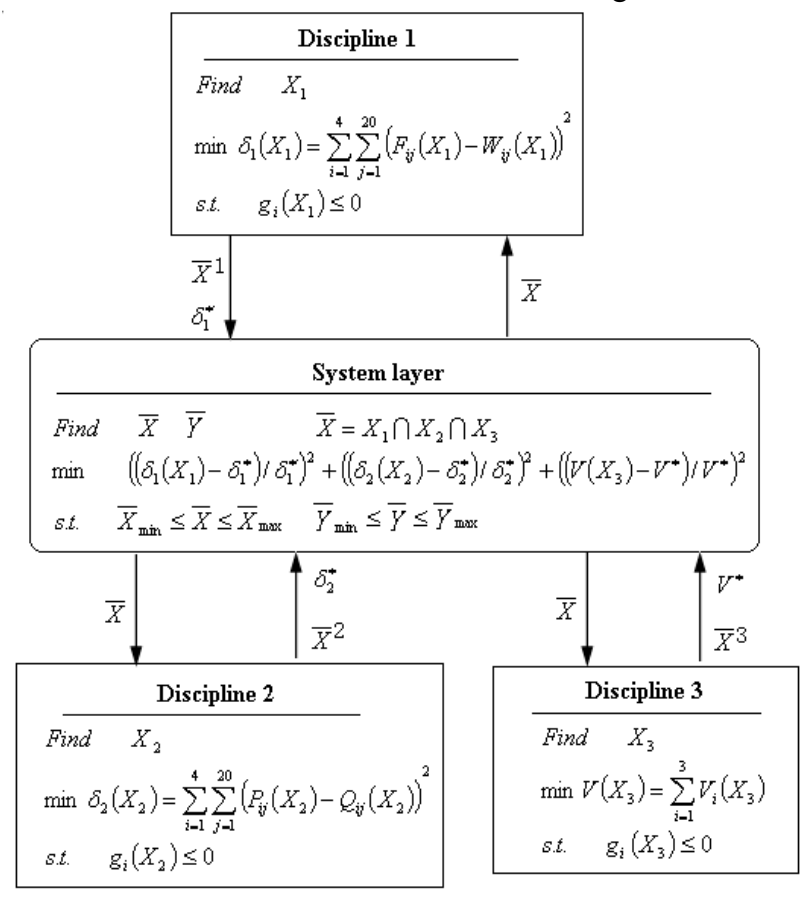

Fig. 6 Three disciplines of the CO model of the excavator backhoe

\section{Sample CO Design of the Backhoe Device}

To test the MDCO design, the excavator in [11] is taken as an example. The initial data regarding the excavator backhoe device are listed in Table 1.

The main parameters of the excavator [11] are as follows: weight is 7.8 tons; bucket capacity is $0.3 \mathrm{~m}^{3}$; the diameter of the boom hydraulic cylinder is $110 \mathrm{~mm}$ and the diameter of its piston rod is $55 \mathrm{~mm}$; and stroke is $835 \mathrm{~mm}$. The diameter of the bucket hydraulic cylinder is $125 \mathrm{~mm}$ and the diameter of its piston rod is $63 \mathrm{~mm}$; stroke is 725 $\mathrm{mm}$; the working pressure of the hydraulic system is 17.5 $\mathrm{MPa}$; and the hydraulic pump flow is $63 \mathrm{l} / \mathrm{min}$.

Using PS-GA, we calculated the MDCO model of the backhoe device. The design results of MDCO, SDO, and the original design are given in Table 2. Simultaneously, the parameterized virtual prototype (VP) of the excavator backhoe device is established in ADAMS, as depicted in Fig. 7. In MDCO, the values of the design variables serve as input parameters to generate the optimized VP. This prototype is called a multidisciplinary virtual prototype (MVP). The process through which MVP was obtained is similar to that undergone by the single discipline virtual prototype (SVP). When the digging of MVP is simulated, the simulation curves of breakout force (M-BFSC) and arm crowd force (M-ACFSC) are generated. When SVP is the digging simulation, the simulation curves of breakout force (S-BFSC) and the arm crowd force (S-ACFSC) are obtained. $\mathrm{M}-\mathrm{BFSC}, \mathrm{S}-\mathrm{BFSC}$, and the resistance to bucket digging are presented in Fig. 8, whereas Fig. 9 displays M-ACFSC, S$\mathrm{ACFSC}$, and the curve of arm digging resistance.

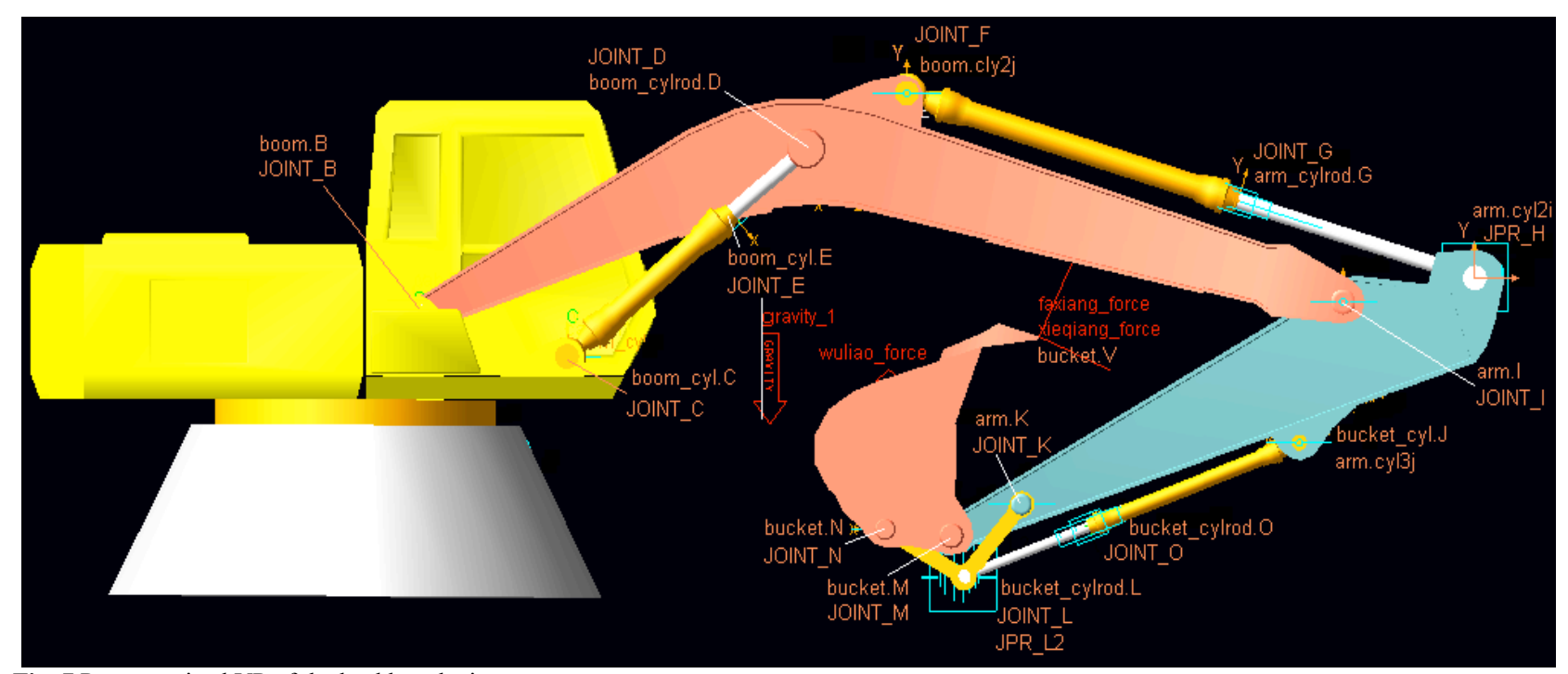

Fig. 7 Parameterized VP of the backhoe device. 


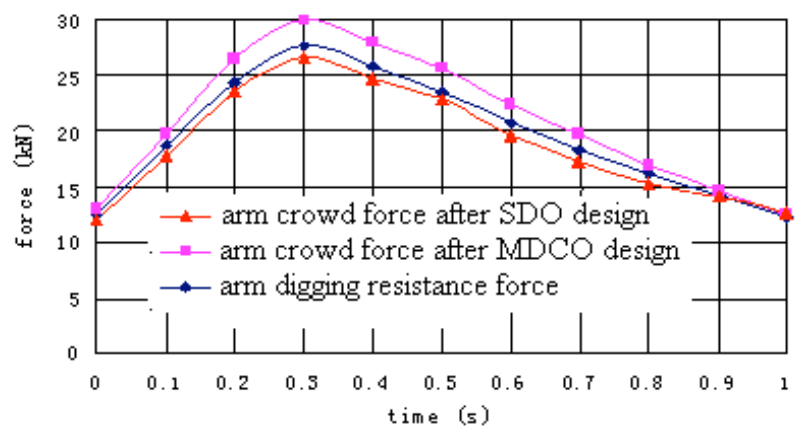

Fig. 8 Curves of arm crowd force and arm digging resistance as obtained with different optimization methods

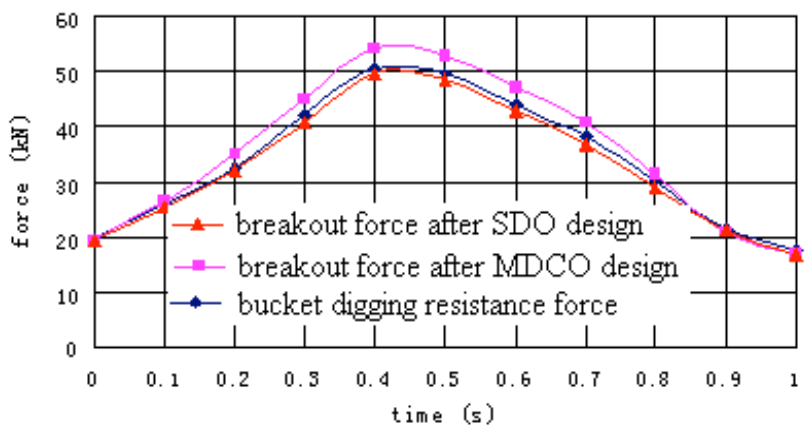

Fig. 9 Curves of breakout force and bucket digging resistance as generated with different optimization methods

Table 1. Initial data on the backhoe device [11] (unit: $\mathrm{mm}$ )

\begin{tabular}{|c|c|c|c|c|c|}
\hline $\begin{array}{l}\text { Parameter } \\
\text { symbol }\end{array}$ & Parameter connotation & $\begin{array}{l}\text { Parameter } \\
\text { value }\end{array}$ & $\begin{array}{l}\text { Parameter } \\
\text { symbol }\end{array}$ & $\begin{array}{l}\text { Parameter } \\
\text { connotation }\end{array}$ & $\begin{array}{l}\text { Parameter } \\
\text { value }\end{array}$ \\
\hline$L_{1}$ & Boom length & 2504 & $L_{7}$ & $|\mathrm{CB}|$ & 1600 \\
\hline$L_{2}$ & Arm length & 1350 & $L_{8}$ & $|\mathrm{DF}|$ & 1550 \\
\hline$L_{1 \min }$ & $\begin{array}{l}\text { Minimum length of the } \\
\text { boom cylinder }\end{array}$ & 1100 & $L_{9}$ & $|\mathrm{EF}|$ & 415.5 \\
\hline$L_{2 \min }$ & $\begin{array}{l}\text { Minimum length of the } \\
\text { arm cylinder }\end{array}$ & 930 & $L_{10}$ & $|\mathrm{FG}|$ & 346 \\
\hline$L_{3 \min }$ & $\begin{array}{l}\text { Minimum length of the } \\
\text { bucket cylinder }\end{array}$ & 1000 & $L_{11}$ & $|\mathrm{EG}|$ & 570 \\
\hline$R_{1 \max }$ & Maximum reach & 5450 & $L_{14}$ & $|\mathrm{NH}|$ & 385 \\
\hline$H_{1 \max }$ & Maximum digging depth & 4300 & $L_{15}$ & $|\mathrm{GN}|$ & 1392 \\
\hline$H_{3 \max }$ & $\begin{array}{l}\text { Maximum dumping } \\
\text { height }\end{array}$ & 3445 & $L_{21}$ & $|\mathrm{NQ}|$ & 199.5 \\
\hline$L_{5}$ & $|\mathrm{CA}|$ & 451 & $L_{29}$ & $|\mathrm{HK}|$ & 357 \\
\hline$L_{6}$ & $|\mathrm{CD}|$ & 1110 & $\overline{-}$ & $\longrightarrow$ & $\longrightarrow$ \\
\hline
\end{tabular}

Table 2. Comparison of the design results of $\mathrm{MDCO}, \mathrm{SDO}$, and the original design.

\begin{tabular}{|c|c|c|c|c|}
\hline & $\begin{array}{c}\text { Maximum arm } \\
\text { crowd force } \\
\text { (Unit: } k N)\end{array}$ & $\begin{array}{l}\text { Maximum } \\
\text { breakout force } \\
\text { (Unit: kN) }\end{array}$ & $\begin{array}{c}\text { Volume of } \\
\text { backhoe device } \\
\left(\text { Unit: } \mathbf{m m}^{* * 3)}\right.\end{array}$ & $\begin{array}{l}\text { Maximum stress } \\
\text { (Unit: MPa) }\end{array}$ \\
\hline $\begin{array}{c}\text { Original } \\
\text { design results [11] }\end{array}$ & 26.8 & 45.8 & $1.38 \times 10 * * 9$ & 227.2 \\
\hline $\begin{array}{l}\text { SDO design } \\
\text { results }[11]\end{array}$ & 28.3 & 45.2 & - & 224.5 \\
\hline MDCO design results & 30.6 & 49.6 & $1.25 \times 10^{* * 9}$ & 223.1 \\
\hline
\end{tabular}

\section{Discussion}

(1)Table 2 shows that the design results of MDCO are optimal, followed by those of SDO and the original design. In the MDCO design, the maximum arm crowd force displayed by the backhoe device was $8.1 \%$ and $14.2 \%$ higher that in the SDO design and in the original design, respectively. A similar trend was observed with respect to maximum breakout force. In the MDCO design, the quality (volume) of the backhoe device was 9.5\% lower than that in the original design, thus indicating the reduced consumption of raw material and economical material manufacturing. Thus, the MDCO design can not only improve the mechanical performance of the backhoe device, but it also reduces raw material cost and enhances the competitiveness of the product.

(2) As shown in Fig. 8, the arm crowd force is much stronger than the force of arm digging resistance during arm excavation when the MDCO design is applied to the backhoe device. Similar results are observed with breakout force, as indicated in Fig. 9. Therefore, MDCO increases digging efficiency and productivity because digging force is enhanced.

The MDCO design technology is suitable for complex systems. It not only organizes complicated systems for optimization, but it also optimizes such systems easily.

\section{Conclusions}

(1) The global optimization design of an excavator working device is realized by using the MDCO method based on the order of "mechanism-load-structure". This method integrates disciplinary optimization with global optimization by considering the interactions among disciplines.

(2) In this study, an improved PSO algorithm called PS-GA is proposed. This algorithm essentially applies the hybrid concept of GA to the PSO algorithm to generate a composite intelligent group algorithm. PS-GA can accelerate 
the evolution speed of a particle swarm and enhance solving efficiency.

(3) The mechanisms and structures of the MDCO model of the excavator backhoe device are established according to MDCO theory. The model is then calculated by using PS-GA.

(4) The values of the MDCO and SDO design variables are used as parameters. The MVP and SVP of the excavator backhoe device simulate digging under similar conditions in ADAMS. The results of excavation simulation suggest that in MVP, arm crowd force is $8.1 \%$ higher than that in SVP under conditions of design power and oil pressure. Similarly, the breakout force in MVP was approximately $8.3 \%$ higher than that in SVP. The quality (volume) of the entire device decreased by $9.5 \%$, and the maximum stress in the main structure changed little.

\section{Acknowledgments}

The authors appreciate the support of the National Natural Science Foundation of China (No.51175086.).

\section{References}

1. Ilan Kroo, Steve Altus, Robert Braun, et al. "Multidisciplinary optimization methods for aircraft preliminary design", Proceedings of the 5th AIAA/USAF/NASA/ISSMO Symposium on Multidisciplinary Analysis and Optimization, Panama, America, Sept. 1994, Vol. 1. AIAA Paper No. 94-4325, pp. 697-707

2. Timothy W. Simpson, Joaquim. R. R. A. Martins. "Multidisciplinary design optimization for complex engineered systems: report from a national science foundation workshop", Journal of Mechanical Design, 133(10), 101002, 2011. pp.1-10.

3. Jong II Yoon, Dinh Quang Truong, Kyoung Kwan Ahn. “A generation step for an electric excavator with a control strategy and verifications of energy consumption", International Journal of Precision Engineering and Manufacturing, 14(5), 2013, pp.755-766.

4. Andrew R. Price, Andy J. Keane, Carren M. E. Holden. "On the Coordination of Multidisciplinary Design Optimization Using Expert Systems", AIAA Journal, 49(8), 2011, pp.1778-1794.

5. SU Ruiyi, GUI Liangjin, WU Zhangbin, et al. "Multidisciplinary Design and Collaborative Optimization for Bus Body", Chinese Journal of Mechanical Engineering, 46(18), 2010, pp.128-133. (In Chinese).
6. Mathieu Balesdent, Nicolas Bérend, Philippe Dépincé, et al. "A survey of multidisciplinary design optimization methods in launch vehicle design", Structural and Multidisciplinary Optimization, 45(5), 2012, pp.619-642.

7. J. Škifić, A. Radošević, D. Brajković, et al. "Numerical simulations of hydraulic transients in hydropower plant Jajce II", Engineering Review, 33(1), 2013, pp.51-56.

8. M. A. Zaman, S. A. Mamun, Md. Gaffar, et al. "Phased array synthesis using modified particle swarm optimization", Journal of Engineering Science and Technology Review, 4(1), 2011, pp.68-73.

9. Mohammad Reza Farmani, Jafar Roshanian, Meisam Babaie, et al. "Multi-objective collaborative multidisciplinary design optimization using particle swarm techniques and fuzzy decision making", Proceedings of the Institution of Mechanical Engineers, Part C: Journal of Mechanical Engineering Science, 226(9), 2012, pp.2281-2295.

10. T.Yalcinoz, H. Altun "Power economic dispatch using a hybrid genetic algorithm", IEEE Power Engineering Review, 21(3), 2001, pp.59-60.

11. SUN zhiguang, "The Optimzing \& Imitation to the Hydraulic Grab Task Device”, Jilin university master's thesis, 2005. (In Chinese) 